\title{
A Calculation Method of Available Transmission Capacity for Medium and Long-term Electricity Trading
}

\author{
Fei Shi ${ }^{1 a^{*}}$, Shuhai Feng ${ }^{1 \mathrm{~b}}$, Hengfeng Zheng ${ }^{1 \mathrm{c}}$, Liwen Wang ${ }^{1 \mathrm{~d}}, \mathrm{Xu} \mathrm{Wu}^{2 \mathrm{e}}$ \\ ${ }^{1}$ Electric Power Research Institute, Nanjing, China \\ ${ }^{2}$ State Grid Anhui Electric Power Company
}

\begin{abstract}
In view of the fact that China's power dispatching agencies and trading centers are relatively independent, but the medium and long-term power transactions need physical execution, which leads to higher requirements for transaction security boundary, this paper proposes a calculation method of available transmission capacity for medium and long-term power transactions, which calculates the available transmission capacity of transmission channels through probabilistic method to improve the enforceability of transaction results. Numerical simulation results also verify the effectiveness of the algorithm.
\end{abstract}

\section{Introduction}

The calculation of available transmission capacity of power system began in the 1970s and has a history of 30 years ${ }^{1}$. In the traditional operation mode of power industry ${ }^{2-4}$, it is mainly used to evaluate the interconnection strength and compare the advantages and disadvantages of different transmission system structures. In the power market environment, the operation conditions of power system become more complex, and economic interests drive market participants to use transmission equipment to the maximum extent $t^{5-7}$. In this context, the calculation of available transmission capacity is very important. It can not only show the security and stability margin of power grid operation, so as to reduce the probability of congestion, but also provide market participants with detailed information of power grid usage, so as to guide them to participate in the market.

The power market model of dispatching and trading integration represented by the United States can consider the system security constraints in the clearing process through security constrained unit commitment and security constrained economic dispatch, so as to accurately coordinate the economic benefits and system security requirements ${ }^{8}$. However, China's trading institutions and dispatching institutions are relatively independent, and the safe operation boundary of the system is determined by the dispatching institutions, while the medium and long-term trading is carried out by the power trading center. Aiming at the situation that the transaction result can not be executed, the optimal reduction of the transaction result is used to make it meet the system security constraints. Transaction reduction is the last line of defense to ensure the security of the system, but frequent transaction reduction is easy to cause the problem of market fairness ${ }^{910}$. Therefore, this paper mainly studies how to calculate the available transmission capacity (ATC) that can be used for further transactions under the security boundary conditions given by the dispatching agency, so as to improve the enforceability of medium and long-term transactions.

\section{ATC Calculation method based on dispatching security boundary}

In the 1990s, North American Electric Reliability Council unified the concept of transmission limit and proposed a detailed definition and calculation framework of available transmission capacity11. This work has been widely recognized in the world.

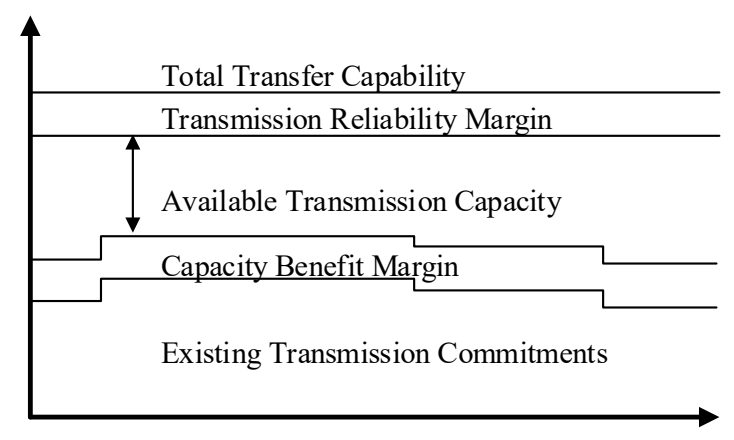

Fig 1 Definition of available transmission capacity

As shown in the figure, ATC refers to the surplus transmission capacity that can be used for further commercial activities in the actual transmission system on the basis of existing agreements. Mathematically, ATC can be expressed as total transfer capacity (TTC) minus transmission reliability margin (TRM), capacity benefit margin $(\mathrm{CBM})$ and existing transmission commitment (ETC)

\footnotetext{
aemail: shifei@epri.sgcc.com.cn, bemail: fengshuhai@epri.sgcc.com.cn,

cemail: zhenghf@epri.sgcc.com.cn, ${ }^{\mathrm{d} e m a i l:}$ wangliwen@epri.sgcc.com.cn

eemail:wux201x@ah.sgcc.com.cn
} 


$$
A T C=T T C-T R M-E T C-C B M
$$

In the formula, TTC is the core problem of traditional ATC calculation, but considering the characteristics of China's electric power medium and long-term trading, the safe operation boundary of the system is determined by the dispatching center. TTC should directly use the value given by the electric power dispatching organization, and the electric power trading center does not need and cannot carry out this calculation. In addition, the calculation of TRM and CBM mostly adopts fixed proportion, so the core problem of ATC calculation is transformed into the calculation of ETC.

The calculation of ETC, that is, the occupancy of existing transaction to the transaction channel, can be calculated by comprehensive sensitivity analysis and transaction power decomposition results. The sensitivity calculation method is as follows.

The deterministic load flow equation in power system can be summarized as:

$$
W=f(X)
$$

$W$ is the node injection vector, including the active power and reactive power, $X$ is the state variable, including the node voltage amplitude and phase angle. The state variable $X_{0}$ can be solved by Newton method under certain injection $W_{0}$

When the node injection changes on the basis of the ground state power flow, it can be expressed as:

$$
W=W_{0}+\Delta W
$$

where $W_{0}$ is the value of expectation, $\Delta W$ is the stochastic disturbance.

By expanding formula(3) with Taylor series, we can get

$$
\begin{aligned}
W & =W_{0}+\Delta W=f\left(X_{0}+\Delta X\right) \\
& =f\left(X_{0}\right)+J_{0} \Delta X+\cdots
\end{aligned}
$$

$J_{0}$ is the Jacobian matrix used in the last iteration of Newton method power flow calculation. Ignoring the higher order term of equation(4), we can get the following results:

$$
\Delta W=J_{0} \Delta X
$$

Thus we'll get:

$$
\Delta X=J_{0}^{-1} \Delta W=S_{0} \Delta W
$$

where $S_{0}$ is the inverse matrix of $J_{0}$, generally it is called sensitivity matrix.

Since active power flow is the main concern in medium and long-term transactions, the branch power flow equation can be linearized. When the state variables of each node are known, the branch power flow equation can be written as follows:

$$
Z=g(X)
$$

The following formula can be obtained by expanding the equation according to Taylor series:

$$
\begin{aligned}
Z & =Z_{0}+\Delta Z=g\left(X_{0}+\Delta X\right) \\
& =g\left(X_{0}\right)+G_{0} \Delta X+\ldots
\end{aligned}
$$

where:

$$
G_{0}=\left.\frac{\partial Z}{\partial X}\right|_{X=X_{0}}
$$

Ignoring the higher order term of equation(8), we can get

$$
\begin{gathered}
\Delta Z=G_{0} S_{0} \Delta W=T_{0} \Delta W \\
\Delta z^{k}=T_{0}^{k} \cdot \Delta w^{k}
\end{gathered}
$$

Through the above formula, the sensitivity relationship between any branch and node in the network can be calculated.

For cross provincial and cross regional transactions, the sensitivity calculation of DC tie line should also be considered. Based on the actual power control mode of the DC tie line, the DC transmission power is only related to the sending end node and the receiving end node connected to the DC tie line, and their sensitivities are 1 and -1 , respectively. The sensitivity distribution factors of the other nodes not connected to the DC tie line are all 0.

$$
Z_{t}=\sum_{i=1}^{I} G_{0} S_{0} W_{t i}
$$

Where $W_{t i}$ is the power decomposition value of the ith transaction at time $t$.

It is worth noting that the medium and long-term trading power curve has the characteristics of large time span and strong uncertainty ${ }^{12}$. In view of this feature, the uncertainty of node power decomposition should be considered in ATC calculation process summary, so as to accurately judge the distribution range of available transmission capacity of actual load of section.

When $W_{t i}$ is no longer a constant, but a probability distribution function, the calculation of equation(12) requires convolution calculation of random variables, and the amount of calculation is large. In order to simplify the calculation process, the probability distribution of random variables can be obtained by introducing the cumulant and Gram-Charlier series expansion method and using the simple algebraic operation of the cumulant.

The k-order semi invariants of random variables $\gamma_{k+1}$ can represented by polynomials which can be expressed by the center moments of random variables. The recurrence relation is as follows:

$$
\begin{gathered}
\gamma_{1}=\alpha_{1} \\
\gamma_{k+1}=\alpha_{k+1}-\sum_{j=1}^{k} C_{k}^{j} \alpha_{j} \gamma_{k-j+1}
\end{gathered}
$$

On the other hand, from the cumulants of each order, the recurrence relation of the central moment of each order is obtained as follows:

$$
\begin{gathered}
\alpha_{1}=\gamma_{1} \\
\alpha_{1+1}=\gamma_{v+1}+\sum_{j=1}^{v} C_{v}^{j} \alpha_{j} \gamma_{v-j+1}
\end{gathered}
$$

The mathematic definitions and descriptions of cumulant method are related to many equations ${ }^{13}$, here we won't list in details. ATC calculation based on cumulant method mainly applies its two characteristics:

(1) The cumulant of the sum of independent random variables is equal to the sum of each random variable's cumulant.

(2) k-order cumulant of $\alpha$ times of random variables is equal to $\alpha^{k}$ times of its k-order cumulant. 
According to these, we can obtain the approaches of getting node power injection's each order cumulant $\Delta w^{k}$ and unknown variable's each order cumulant $\Delta x^{k}$ :

$$
\begin{aligned}
& \Delta w^{k}=\Delta w_{G}^{k}+\Delta w_{L}^{k} \\
& \Delta x^{k}=S_{0}^{k} \cdot \Delta w^{k}
\end{aligned}
$$

In the equations, $\Delta w_{G}^{k}$ and $\Delta w_{L}^{k}$ stand for k-order cumulants of seller's and the buyer's injection power.

After obtaining $\Delta Z_{t}^{k}$ of branch power, the distribution function of $\Delta Z_{t}$ can be obtained by the relationship between the cumulant and the center distance and the method of Gram-Charlier series expansion ${ }^{14}$. The random distribution of branch power can be obtained by formula(17).

$$
f(x)=\varphi(x)+\frac{A_{1} \varphi^{\prime}(x)}{1 !}+\frac{A_{2} \varphi^{\prime \prime}(x)}{2 !}+\frac{A_{3} \varphi^{(3)}(x)}{3 !}+\frac{A_{4} \varphi^{(4)}(x)}{4 !}+\ldots
$$

In this equation, $\varphi(x)$ is the probability density function of the standard normal distribution, and the coefficient $A_{i}$ can be obtained from formula(18), where $\delta$ is the standard deviation of the random variable. $\varphi^{(k)}(x)$ can be obtained by Hermite polynomial formula $(19)^{15}$.

$$
\begin{gathered}
A_{1}=0, \quad A_{2}=0, \quad A_{3}=-\frac{\Delta Z_{t}^{3}}{\delta^{3}}, \quad A_{4}=\frac{\Delta Z_{t}^{4}}{\delta^{4}}, \quad A_{5}=-\frac{\Delta Z_{t}^{5}}{\delta^{5}}, \\
A_{6}=\frac{\Delta Z_{t}^{6}}{\delta^{6}}+10\left(\frac{\Delta Z_{t}^{3}}{\delta^{3}}\right)^{2}, \quad A_{7}=-\frac{\Delta Z_{t}^{7}}{\delta^{7}}+35 \frac{\Delta Z_{t}^{3}}{\delta^{3}} \frac{\Delta Z_{t}^{4}}{\delta^{4}} \\
A_{8}=\frac{\Delta Z_{t}^{8}}{\delta^{8}}+56 \frac{\Delta Z_{t}^{3}}{\delta^{3}} \frac{\Delta Z_{t}^{5}}{\delta^{5}}+35\left(\frac{\Delta Z_{t}^{4}}{\delta^{4}}\right)^{2} \\
\varphi^{(k)}(x)=(-1)^{k} \varphi(x) H_{k}(x), k=0,1, \ldots
\end{gathered}
$$

Where, the coefficient $\mathrm{H}$ is calculated as follows:

$H_{0}(x)=1, \quad H_{1}(x)=x, \quad H_{2}(x)=x^{2}-1, \quad H_{3}(x)=x^{3}-3 x$

$H_{4}(x)=x^{4}-6 x^{2}+3, \quad H_{5}(x)=x^{5}-10 x^{3}+15 x$

$H_{6}(x)=x^{6}-15 x^{4}+45 x^{2}-15$

$H_{7}(x)=x^{7}-21 x^{5}+105 x^{3}-105 x$

$H_{8}(x)=x^{8}-28 x^{6}+210 x^{4}-420 x^{2}+105 n$

Thus, the probability distribution function of ETC on each branch or transmission channel can be calculated.

\section{Case studies}

Taking IEEE118 system as an example, there are 19 nodes generating output in this example. For simplicity, the power decomposition result of all nodes is $230 \mathrm{MW}$, the upper limit of branch active power is $350 \mathrm{MW}$, and the total power of TRM and CBM is $50 \mathrm{MW}$. When the uncertainty of trading capacity is not considered, the order of available transfer capacity of each branch is as follows:

Table 1 ATC calculation results without considering the uncertainty of node injection power

\begin{tabular}{|c|c|c|c|c|}
\hline Branch number & Begin Node & End Node & Active Power & ATC \\
\hline 9 & 9 & 10 & 228.76 & 71.24 \\
\hline 7 & 8 & 9 & 227.35 & 72.65 \\
\hline 176 & 110 & 111 & 217.60 & 82.40 \\
\hline 134 & 86 & 87 & 214.43 & 85.57 \\
\hline 38 & 26 & 30 & 213.30 & 86.70 \\
\hline 51 & 38 & 37 & 200.78 & 99.22 \\
\hline 8 & 8 & 5 & 197.62 & 102.38 \\
\hline 104 & 65 & 68 & 186.83 & 113.17 \\
\hline 183 & 68 & 116 & 184.13 & 115.87 \\
\hline 133 & 85 & 86 & 177.24 & 122.76 \\
\hline
\end{tabular}

Table 1 lists the 10 branches with the smallest ATC value, which are the main bottleneck channels affecting the transaction.

If we further consider the uncertainty of the decomposition of the trading power, and assume that the power injection of the generating nodes obeys the normal distribution of $\mathrm{N}(230,46)$, the probability of branch power flow out of limit is considered with $90 \%$ confidence. On this basis, the available transmission capacity of each branch is calculated as follows.

Table 2 ATC calculation results considering the uncertainty of node injection power

\begin{tabular}{|c|c|c|c|c|c|}
\hline $\begin{array}{c}\text { Branch } \\
\text { number }\end{array}$ & Begin Node & End Node & $\begin{array}{c}\text { Active Power } \\
\text { Expectation }\end{array}$ & $\begin{array}{c}\text { Active Power } \\
\text { Variance }\end{array}$ & ATC \\
\hline 104 & 65 & 68 & 186.83 & 100.45 & -15.40 \\
\hline 9 & 9 & 10 & 228.76 & 45.51 & 12.99 \\
\hline 7 & 8 & 9 & 227.35 & 45.06 & 14.98 \\
\hline
\end{tabular}




\begin{tabular}{|c|c|c|c|c|c|}
\hline 176 & 110 & 111 & 217.60 & 41.08 & 29.81 \\
\hline 134 & 86 & 87 & 214.43 & 39.08 & 35.55 \\
\hline 38 & 26 & 30 & 213.30 & 33.91 & 43.30 \\
\hline 8 & 8 & 5 & 197.62 & 26.59 & 68.34 \\
\hline 133 & 85 & 86 & 177.24 & 31.25 & 82.76 \\
\hline 51 & 38 & 37 & 200.78 & 6.03 & 91.50 \\
\hline 163 & 100 & 103 & 143.08 & 41.75 & 103.49 \\
\hline
\end{tabular}

Based on the calculation results in Table 1 and table 2, it can be seen that whether the uncertainty of the decomposition result of trading power is considered has a great influence on the calculation result of ATC in trading channel.

In the process of actual transaction execution, in order to take into account the impact of the uncertainty of longterm electricity trading, the limit value is generally discounted according to a certain proportion. According to the basic rules for medium and long term electricity trading, the annual trading limit shall be no less than $80 \%$ of the available transmission capacity of key channels, while the monthly trading and intra month trading shall be $90 \%$ and $95 \%$ respectively. This method is simple and clear. It can deal with the uncertainty of all nodes and branches according to a unified standard, but it may not be able to accurately consider the different distribution characteristics of different channels.

Table 3 difference of ATC calculation results of two different treatment methods

\begin{tabular}{|c|c|c|c|c|c|c|c|}
\hline \multirow{2}{*}{ Begin Node } & \multirow{2}{*}{ End Node } & \multicolumn{2}{|c|}{ Deterministic calculation method } & \multicolumn{3}{|c|}{ Probability calculation method } \\
\cline { 3 - 8 } & & $95 \%$ Limit & $90 \%$ Limit & $80 \%$ Limit & $5 \%$ Variance & $10 \%$ Variance & 20\%Variance \\
\hline 9 & 10 & 53.74 & 36.24 & 1.24 & 56.68 & 42.12 & 12.99 \\
\hline 8 & 9 & 55.15 & 37.65 & 2.65 & 58.23 & 43.82 & 14.98 \\
\hline 110 & 111 & 64.9 & 47.4 & 12.4 & 69.25 & 56.10 & 29.81 \\
\hline 86 & 87 & 68.07 & 50.57 & 15.57 & 73.07 & 60.56 & 35.55 \\
\hline 26 & 30 & 69.2 & 51.7 & 16.7 & 75.85 & 65.00 & 43.30 \\
\hline 38 & 37 & 81.72 & 64.22 & 29.22 & 97.29 & 95.36 & 91.50 \\
\hline 8 & 5 & 84.88 & 67.38 & 32.38 & 93.87 & 85.36 & 68.34 \\
\hline 65 & 68 & 95.67 & 78.17 & 43.17 & 81.03 & 48.89 & -15.40 \\
\hline 68 & 116 & 98.37 & 80.87 & 45.87 & 115.87 & 115.87 & 115.87 \\
\hline 85 & 86 & 105.26 & 87.76 & 52.76 & 112.76 & 102.76 & 82.76 \\
\hline
\end{tabular}

It can be seen from the calculation results in Table 3 that the traditional calculation method of limit discount according to the unified standard for most branches is more consistent with the method proposed in this paper, but the uncertainty characteristics of different branches are quite different, and the traditional calculation method can not well understand the probability distribution characteristics of all branches, which may cause some transactions to be unable to be executed and may need to be implemented Make adjustments.

\section{Conclusion}

China's annual planned electricity and bilateral trading electricity need physical delivery, and the power dispatching agency and trading center are relatively independent, so it is difficult for trading center to carry out medium and long-term trading. In this paper, a calculation method of available transmission capacity is proposed, which is suitable for medium and long-term electricity trading. The effectiveness of the algorithm is verified by numerical simulation. It is important to note that the probability calculation method is slightly deviated from the traditional Chinese power dispatching control concept. How to apply the probabilistic analysis control method in the field of power transaction and dispatching needs further exploration in the follow-up research process.

\section{Acknowledgments}

Project Supported by STATE GRID Corporation of China; the algorithm research and development of long-term transactions of ATC for large-scale and complex network.

\section{References}

1. Landgren G L, Anderson S W. Simultaneous power interchange capability analysis[J]. IEEE Trans on Power Apparatus and Systems, 1973,92(6):19731986.

2. Ejebe G C, Waight J G, Sanots-Nieto M, Tinney W F. Fast Calculation of Linear Available Transfer Capability[J]. IEEE Transactions on Power Systems, 2000,15(3):1112-1116. 
3. Dai Youjie, Mc Calley J D, Vittal V. Simplification, Expansion and Enhancement of Direct Interior Point Algorithm for Power System Maximum Load ability[J]. IEEE Transactions on Power Systems, 2000,15(3):1014-1021.

4. Wang Feng, Bai Xiaomin . OPF based transfer capability calculation[J].Proceedings of the CSEE, 2002,22(11):35-39.

5. Li Zhu, Pang Bo, Li Guodong, et al. Development of unified european electricity market and its implications for China[J].Automation of Electric Power Systems, 2017,41(24):2-9.

6. Greene S, Dobson I, Alvarado F L, Sensitivity of Transfer Capability Margins with a Fast Formula[J]. IEEE Transactions on Power Systems, 2002,17(1):34-40.

7. Ramezani M. Haghifam M. R, Singh C, et al. Determination of capacity benefit margin in multi area power systems using particle swarm optimization. IEEE Transactions on Power Systems, 2009,24(2):631-641.

8. Wei Fen.Experiences in PJM market in the United States[J]. Automation of Electric Power Systems, 2003,27(8):32-35

9. Liu Dunnan, Wu Yaguang, Jiang Xiaoliang, et al. Impact of security constraints on regional electricity market[J].Automation of Electric Power Systems, 2005,29(21):9-13.

10. Shu Chang, Zhong Haiwang, Xia Qing, et al. Monthly electricity market design based on constraint relaxation[J].Proceedings of the CSEE, 2016,36(3):587-595

11. Federal Energy Regulatory Commission. Open Access Same-Time Information System and Standards of Conduct. Docket No. RM 95-9-000, Order 889, April 1996.

12. Che Xingru, Bai Guohua, Li Guoqing, et al. Evaluation of available transmission capacity for hybrid AC/DC system considering the uncertain factors[J].Journal of Northeast Dianli University, 2014,34(1):52-58.

13. Wang Xifan, Wang Xiuli. Probabilistic load flow analysis in power system [J]. Journal of Xi'an Jiaotong University, 1988,22(3):87-97.

14. Shi Dong-yuan, Cai De-fu, Chen Jin-fu, et al. Probabilistic load flow calculation based on cumulant method considering correlation between input variables $[\mathrm{J}]$. Proceedings of the CSEE, 2012,32(28):104-113

15. ZHANG Pei, Lee $S$ T. Probabilistic load flow computation using the method of combined cumulants and Gram-Charlier expansion [J]. IEEE Trans on Power Systems, 2004,19(1):676-682. 